\title{
Ibuprofen versus fosfomycin for uncomplicated urinary tract infection in women: randomised controlled trial
}

\author{
Ildikó Gágyor, ${ }^{1} J u t t a ~ B l e i d o r n,{ }^{2}$ Michael M Kochen, ${ }^{3}$ Guido Schmiemann, ${ }^{4}$ Karl Wegscheider, ${ }^{5}$ \\ Eva Hummers-Pradier ${ }^{1}$
}

${ }^{1}$ Department of General

Practice, University Medical

Center Göttingen,

Humboldtallee 38, 37073

Göttingen, Germany

2Institute of General Practice,

Hannover Medical School,

Carl-Neuberg-Strasse 1, 30625

Hannover, Germany

3Department of Medicine,

Division of General Practice,

University Medical Centre,

Elsässerstrasse 2m, 79110

Freiburg, Germany

${ }^{4}$ Institute for Public Health and Nursing Research, Department

for Health Services Research,

University of Bremen, Grazer

Strasse 4, 28359 Bremen,

Germany

5Department of Medical

Biometry and Epidemiology,

University Medical Centre

Hamburg-Eppendorf,

Martinistrasse 52, 20246

Hamburg, Germany

Correspondence to: I Gágyor

igagyor@gwdg.de

Cite this as: BMJ 2015;351:h6544

http://dx.doi.org/10.1136/bmj.h6544

Accepted: 29 October 2015

\begin{abstract}
STUDY QUESTION

Can treatment of the symptoms of uncomplicated urinary tract infection (UTI) with ibuprofen reduce the rate of antibiotic prescriptions without a significant increase in symptoms, recurrences, or complications? METHODS

Women aged 18-65 with typical symptoms of UTI and without risk factors or complications were recruited in $42 \mathrm{German}$ general practices and randomly assigned to treatment with a single dose of fosfomycin $3 \mathrm{~g}(\mathrm{n}=246$; 243 analysed) or ibuprofen $3 \times 400 \mathrm{mg}(\mathrm{n}=248 ; 241$ analysed) for three days (and the respective placebo dummies in both groups). In both groups additional antibiotic treatment was subsequently prescribed as necessary for persistent, worsening, or recurrent symptoms. The primary endpoints were the number of all courses of antibiotic treatment on days 0-28 (for UTI or other conditions) and burden of symptoms on days 0-7. The symptom score included dysuria, frequency/ urgency, and low abdominal pain.
\end{abstract}

\section{STUDY ANSWER AND LIMITATIONS}

The 248 women in the ibuprofen group received significantly fewer course of antibiotics, had a significantly higher total burden of symptoms, and more had pyelonephritis. Four serious adverse events occurred that lead to hospital referrals; one of these was potentially related to the trial drug. Results have to be interpreted carefully as they might apply to women with mild to moderate symptoms rather than to all those with an uncomplicated UTI.

WHAT THIS PAPER ADDS

Two thirds of women with uncomplicated UTI treated symptomatically with ibuprofen recovered without any antibiotics. Initial symptomatic treatment is a possible

\section{WHAT IS ALREADY KNOWN ON THIS TOPIC}

Women with uncomplicated urinary tract infection are usually treated with antibiotics

It is unknown if symptomatic treatment is a suitable alternative approach with regard to symptoms and to what extent such treatment can reduce the overall antibiotic prescription rate

\section{WHAT THIS STUDY ADDS}

Compared with fosfomycin, initial treatment with ibuprofen substantially reduced antibiotic use in women aged 18-65 with mild to moderate symptoms of urinary tract infection but was less effective for symptom relief, and there were more cases of pyelonephritis

This treatment regimen can be discussed with women who are willing to avoid antibiotics or to accept a delayed prescription approach to be discussed with women willing to avoid immediate antibiotics and to accept a somewhat higher burden of symptoms.

FUNDING, COMPETING INTERESTS, DATA SHARING German Federal Ministry of Education and Research (BMBF) No 01KG1105. Patient level data are available from the corresponding author. Patient consent was not obtained but the data are anonymised and risk of identification is low.

TRIAL REGISTRATION NO

ClinicalTrialGov Identifier NCT01488955.

\section{Introduction}

Uncomplicated urinary tract infections are common in many clinical settings but especially in general practice, where they account for $25 \%$ of antibiotic prescriptions. ${ }^{12}$ Prescription of antibiotics when many cases are self limiting contributes to increased resistance rates, posing a serious health threat. ${ }^{34}$ Disease burden and treatment costs have to be considered as well. ${ }^{5}$ Escherichia coli, the main causative agent, is increasingly resistant to current antibiotics, ${ }^{6}$ and the number of new antibiotics being developed is declining. Many current primary care guidelines, however, still recommend antibiotics as a first line treatment, ${ }^{78}$ at least when standard measures do not lead to symptom relief. As urinary tract infection is often self limiting, ${ }^{9-11}$ and less antibiotic prescribing lowers levels of antibiotic resistance, ${ }^{12-15}$ efforts should be made to reduce rates of prescription.

Earlier trials assessed placebo compared with antibiotic treatment for urinary tract infection as well as delayed prescription of antibiotics. ${ }^{91116}$ These trials reported a delayed resolution of symptoms and prolonged time to bacterial clearance but no serious complications. From qualitative studies and surveys, we know that many affected women are aware of the potential disadvantages of antibiotics and might agree to avoid or postpone such treatment. ${ }^{17-19}$

In a pilot study we compared symptomatic (ibuprofen) with antibiotic treatment (ciprofloxacin) in 79 women with uncomplicated urinary tract infection. Although this study was inadequately powered for a definitive result, it showed that ibuprofen was not inferior for symptom resolution, with 24/36 women recovering without antibiotic treatment. ${ }^{10}$ In the current study we assessed whether the number of antibiotic prescriptions issued for uncomplicated urinary tract infection can be reduced by symptomatic treatment with ibuprofen, reserving antibiotics for women who return with worsening or recurrent symptoms, and without an increase in symptom burden, recurrences, or complications. 


\section{Methods}

Trial design and participants

ICUTI (Immediate versus Conditional treatment of Urinary Tract Infection) was a double blind randomised multicentre comparative effectiveness trial with two parallel active treatment arms (ibuprofen and fosfomycin). The study was conducted according to the Good Clinical Practice Guidelines and the declaration of Helsinki. Safety data were monitored throughout and assessed every six months by the data and safety monitoring board. Serious adverse events were defined as adverse events leading to admission to hospital, disability, permanent damage, or risk of dying or death.

The detailed study protocol has been published elsewhere. ${ }^{20}$ From February 2012 to February 2014 practice staff and general practitioners in 42 general practices in northern Germany systematically approached women aged 18-65 with typical symptoms of urinary tract infection for consent. The upper age limit was chosen as until 2013 fosfomycin was approved only for patients up to age 65.

The study was designed as a pragmatic trial in a general practice setting. ${ }^{21}$ Inclusion criteria were dysuria and/or frequency/urgency of micturition, with or without lower abdominal pain. The main exclusion criteria were any signs of upper urinary tract infection (fever, loin tenderness); current conditions that could increase the likelihood of potentially complicated courses (such as pregnancy or renal diseases); urinary tract infection within the past two weeks; and urinary catheterisation. We also excluded patients currently treated with non-steroidal drugs for pain or antibiotics and those with a history of gastrointestinal ulcers or severe acute or exacerbated chronic conditions. ${ }^{20}$ Dipstick tests and urine culture were performed, but the results did not affect eligibility.

Participating general practitioners were trained in good clinical practice to ensure patient safety and data quality. In addition to electronic database monitoring, an independent monitor (IFS Göttingen) made one on-site monitoring visit per practice and study nurses from the academic teams made further quality management visits to ensure correct documentation. No major or safety problems were detected; some minor corrections, for example completion of missing data, were instigated and problems with screening failures were discussed.

\section{Randomisation and masking}

We used a computerised random number generator to carry out randomisation in blocks of six in a 3:3 ratio. The trial biometrician was not involved in patient recruitment and data collection. The block-wise randomisation implied stratification by site.

An independent pharmacy identically prepared and packed drug units, which were labelled with a code number from the random list. Practices were supplied with packs of six blinded drug units. At inclusion, general practitioners assigned the code number from the drug unit to the patient and all patient related data. Practice teams as well as academic study teams were blinded to allocation and had no access to the random list. For emergency unblinding, sealed opaque envelopes for each random number were kept in the investigator site files, and a random list was kept in the trial pharmacy.

\section{Procedures}

The practice teams were asked to assess all women presenting with symptoms of acute urinary tract infection consecutively for inclusion and exclusion criteria. Eligible women completed a questionnaire to score severity of symptoms and impairment of activity. They also provided a urine sample for dipstick, culture, and pregnancy tests. To assess bias and external validity of study findings, symptom questionnaires were also collected from women who declined to take part in the trial or had exclusion criteria. Participating women were handed out the blinded trial drug package containing either ibuprofen tablets $(3 \times 400 \mathrm{mg}$ daily for three days plus $1 \times 1$ sachet placebo granules) or fosfomycin-trometamol $(1 \times 3 \mathrm{~g}$ sachet plus $3 \times 3$ placebo tablets for three days), according to randomisation. After inclusion, women were asked to start drug treatment themselves. Drug packages had to be returned and were checked for residual drugs.

Women were advised to consult their general practitioners again if symptoms persisted or worsened. In this case, antibiotic treatment was initiated at the discretion of the general practitioner on the basis of the results of urine culture from the baseline visit. Participants received an emergency card providing the telephone number of the trial pharmacy in case emergency unblinding was required. Women scored their daily symptoms and activity impairment in a diary. Study nurses collected data on symptoms as well as drug intake and further antibiotic prescriptions by telephone calls on day $1,3,5$, and 7 , or until recovery. On day 28 , data on antibiotic prescriptions, adverse events, and recurrent urinary tract infections were also collected. All data were recorded into a web based data entry system.

One central laboratory in Göttingen performed all urine cultures. The cut off for positive culture was bacterial count $>10^{2} \mathrm{cfu} / \mathrm{mL}$. Susceptibility test were performed by disk diffusion according to DIN 58940 and DIN 58959 with European Committee on Antimicrobial Susceptibility Testing breakpoints. ${ }^{22}$

\section{Outcomes}

Two co-primary endpoints examined both benefits and risks: the total number of courses of antibiotics on days 0-28 (for urinary tract infection or other conditions) 223 and burden of symptoms on days 0-7, measured as area under the curve of the sums of daily symptom scores. The symptom score included dysuria, frequency/ urgency of micturition, and low abdominal pain, each on a five point scale from 0 (not at all) to 4 (strong/ frequent). ${ }^{10}$ The area under the curve of symptom sum scores has been used before in other trials to measure overall severity and duration of symptoms. ${ }^{2425}$ The trial was to be considered as having a positive outcome if superiority in the first and non-inferiority in the second co-primary endpoint could be proved.

Secondary outcomes were the numbers of severe adverse events, complications (febrile urinary tract infec- 
tion, pyelonephritis, septic syndrome), all adverse events, relapses (recurrent urinary tract infection after initial resolution of symptoms) up to day 28 and within six and 12 months, ${ }^{7}$ and women without symptoms at days four and seven (defined as a symptom sum score of 0 ); symptom load until day four and symptom load with regard to specific symptoms until day seven (specified in the trial protocol as symptom burden until day four, related to each of the three symptoms until day seven ${ }^{7}$ ), assessment of activity impairment on days 1-7, measured with a five item score (range 0-4), referring to the time during which a woman's work or personal activities had been impaired because of urinary tract infection ${ }^{26}$; and the number of daily defined doses of antibiotics per patient (the latter will be reported in a separate paper). As the wording in the trial registry was not as specific as in the protocol, we use in this paper terms and definitions for outcomes as given in the trial protocol. To determine whether there were differences within the groups based on the results of the urine cultures, we performed a post hoc outcome analysis stratified on the basis of positive or negative results on urine culture. After publication of the protocol but before unblinding the data we decided to split the early follow-up period regarding recurrent urinary tract infections into two periods according to the German urinary tract infection guideline $^{7}$ : early relapse of symptoms (recurrence of symptoms up to day 14, after initial resolution) and recurrence of urinary tract infection after initial resolution from day 15 up to day 28. Rates of recurrent urinary tract infection within the prolonged follow-up at six and 12 months will be reported separately once the follow-up is finished.

\section{Statistical analysis}

The sample size calculated for both co-primary outcomes was driven by the non-inferiority part of the trial. If we assume a coefficient of variation of $80 \%$, we would require a sample size of $2 \times 210=420$ evaluable

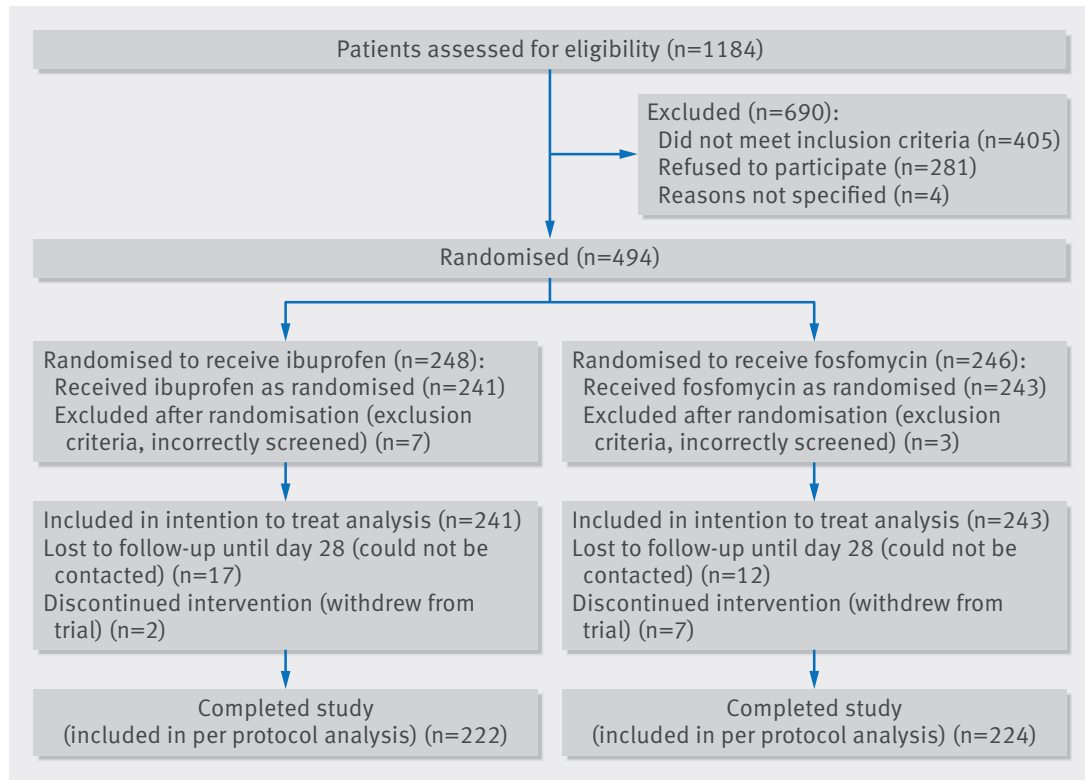

Fig 1 | Flow of participants through trial of ibuprofen versus fosfomycin for women with urinary tract infection patients to reach a power of $90 \%$ for the proof of non-inferiority in case of equivalence of the two study arms (one sided $\alpha=0.025$ ). ${ }^{27}$ As we assumed a dropout rate of $15 \%$, we needed to randomise 494 women to achieve the sample size of 420 patients in the per protocol population. ${ }^{20}$

The primary analysis consisted of two statistical tests as described in the study protocol..$^{20}$ The first co-primary endpoint-"number of courses of antibiotic treatment"-was tested for superiority of the ibuprofen group with an exact Mann-Whitney rank sum test with a one sided test level of $2.5 \%$. The second co-primary endpoint was tested for non-inferiority of ibuprofen compared with fosfomycin by calculating a two sided 95\% confidence interval for the ratio of the symptom burden of the ibuprofen and fosfomycin groups based on a covariance analysis of the log symptom burden with adjustment for baseline log symptom sum score. Non-inferiority was assumed if the total confidence interval was below the non-inferiority margin of $125 \%$. As no standard was available for this kind of study, we followed the suggested margins for bioequivalence studies, which accept levels of $80-120 \%$ of the reference as bioequivalent. We analysed the first co-primary endpoint in the intention to treat population, which consisted of all randomised patients with at least one report on use or non-use of antibiotics. The second co-primary endpoint was analysed in the per protocol population, which consisted of all intention to treat patients with complete seven day follow-up symptom score and subsequently in the intention to treat population. In this population, we had to impute a small number of missing values to calculate the area under the curves. For this purpose we applied an expectation-maximisation algorithm to the available score data to estimate means and covariances based on a normality assumption and chose imputations that maximise the likelihood. As the differences to the analysis of the per protocol population were small, we have presented both endpoints in the same intention to treat population for direct comparability.

The secondary endpoints were analysed with exact Mann-Whitney U rank sum tests and $\chi^{2}$ tests at two sided $\alpha=0.05$ without adjustment for multiplicity or analogous to the second co-primary endpoint.

\section{Patient involvement}

No patients were involved in designing the study or explicitly setting outcome measures; however, outcomes were chosen to reflect daily practice and patient preferences described in earlier studies. ${ }^{1819}$ Preliminary results were disseminated to study participants through their general practitioners. A lay information flyer with final results will be sent to all participating practices and can be used to inform patients with urinary tract infections.

\section{Results}

\section{Randomisation and baseline characteristics}

A total of 1184 women with suspected urinary tract infection were screened, 779 were considered eligible, and 494 were randomly assigned to the treatment or control groups (fig 1). On average, 12 women per 
practice were included in 42 practices.Ten women with exclusion criteria had been included incorrectly by general practitioners and were therefore excluded immediately after recruitment by a masked researcher. The intention to treat population comprised 241 women in the ibuprofen group and 243 in the fosfomycin group. Three women, in whom exclusion criteria were detected later after inclusion, remained in this population. Two women in the ibuprofen group and seven in the fosfomycin group refused further participation and withdrew from the trial.

There were no major differences in baseline characteristics between both groups (table 1). Minor differences concerned duration of symptoms (110 (46\%) women in the fosfomycin group versus 87 (36\%) in the ibuprofen group had symptoms for more than two days) and recurrent urinary tract infections being reported more often by women assigned to the fosfomycin group (54 (23\%) versus $42(17 \%))$.

\section{Primary outcomes}

The number of courses of antibiotic treatment within 28 days was significantly lower in the ibuprofen group. The number of all courses in the fosfomycin group was 283 (243 as part of the study plus 34 courses prescribed additionally for urinary tract infection and six courses prescribed for other reasons, such as bronchitis and otitis media) compared with 94 in the ibuprofen group (81 for

Table 1 | Baseline characteristics of women with uncomplicated urinary tract infection randomised to ibuprofen or fosfomycin. Figures are numbers of women (percentage) unless stated otherwise

\begin{tabular}{|c|c|c|}
\hline & Ibuprofen $(n=241)$ & Fosfomycin $(n=243)$ \\
\hline Mean (SD) age (years) & $37.3(14.6)$ & $37.3(14.3)$ \\
\hline Median (IQR) age (years) & $36.0(24.0-50.0)$ & $34.0(24.0-49.0)$ \\
\hline \multicolumn{3}{|l|}{ Duration of symptoms at inclusion (days)*: } \\
\hline$<1$ & $50(21)$ & $49(21)$ \\
\hline $1-2$ & $104(43)$ & $80(34)$ \\
\hline$>2-7$ & $66(27)$ & $82(34)$ \\
\hline$>7$ & $21(9)$ & $28(12)$ \\
\hline \multicolumn{3}{|l|}{ Symptoms at inclusion*: } \\
\hline Dysuria & $224(93)$ & $218(91)$ \\
\hline Frequency/urgency & $231(96)$ & $232(97)$ \\
\hline Low abdominal pain & $172(71)$ & $169(71)$ \\
\hline Mean (SD) symptom severity sum scoret & $6.0(2.2)$ & $6.1(2.5)$ \\
\hline Median (IQR) symptom severity sum score & $6.0(4.0-8.0)$ & $6.0(4.0-8.0)$ \\
\hline Mean (SD) dysuria score $\neq$ & $2.3(1.0)$ & $2.3(1.1)$ \\
\hline Median (IQR) dysuria score $\ddagger$ & $3.0(2.0-3.0)$ & $2.0(1.0-3.0)$ \\
\hline Mean (SD) frequency/urgency score $\neq$ & $2.4(1.1)$ & $2.4(1.1)$ \\
\hline Median (IQR) frequency/urgency score $\neq$ & $2.0(2.0-3.0)$ & $2.0(2.0-3.0)$ \\
\hline Mean (SD) low abdominal pain score $\ddagger$ & $1.3(1.1)$ & $1.4(1.1)$ \\
\hline Median (IQR) low abdominal pain score $\neq$ & $1.0(0.0-2.0)$ & $1.0(0.0-2.0)$ \\
\hline Recurrent UTI§ & $42(17)$ & $54(23)$ \\
\hline Mean (SD) activity impairment scoreq & $9.6(5)$ & $8.9(6)$ \\
\hline \multicolumn{3}{|l|}{ Dipstick results ${ }^{\star \star}$} \\
\hline Leukocytes positive & $205(85)$ & $200(83)$ \\
\hline Erythrocytes positive & $180(75)$ & $189(78)$ \\
\hline Nitrite positive & $53(22)$ & $46(19)$ \\
\hline \multicolumn{3}{|l|}{ Culture results: } \\
\hline Urine culture positivet† & 179/237 (76) & $181 / 234(77)$ \\
\hline E coli & $143 / 179(80)$ & 142/181 (79) \\
\hline Proteus mirabilis & $12 / 179(7)$ & $8 / 181(4)$ \\
\hline Staphylococcus saprophyticus & $8 / 179(5)$ & $8 / 181(4)$ \\
\hline Enterococcus faecalis & $3 / 179(2)$ & $8 / 181(4)$ \\
\hline Streptococcus agalactiae & $0 / 179(0)$ & $2 / 181(1)$ \\
\hline Klebsiella pneumoniae & 3/179 (2) & $1 / 181(1)$ \\
\hline Other uropathogens & $10 / 179(6)$ & $9 / 181(5)$ \\
\hline Not specified & $0 / 179(0)$ & $3 / 181(2)$ \\
\hline \multicolumn{3}{|l|}{ Susceptibility to fosfomycin (rate): } \\
\hline All uropathogens & 168/181 (93) & $162 / 177(92)$ \\
\hline E coli & 142/143 (99) & $142 / 142(100)$ \\
\hline \multicolumn{3}{|c|}{ 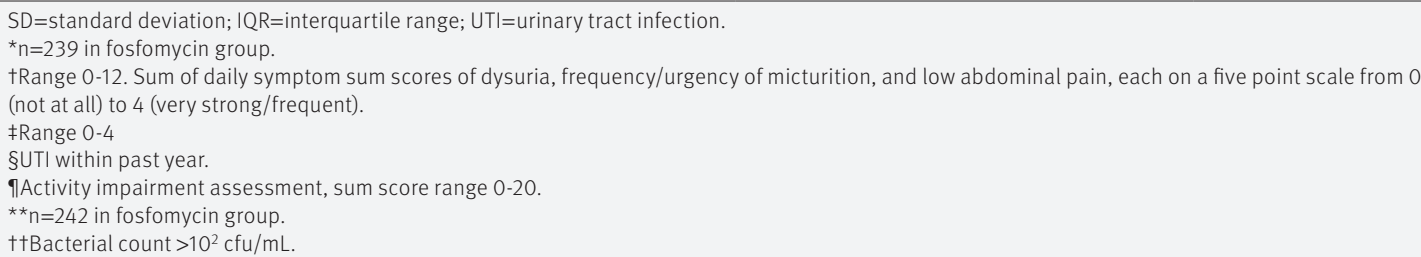 } \\
\hline
\end{tabular}




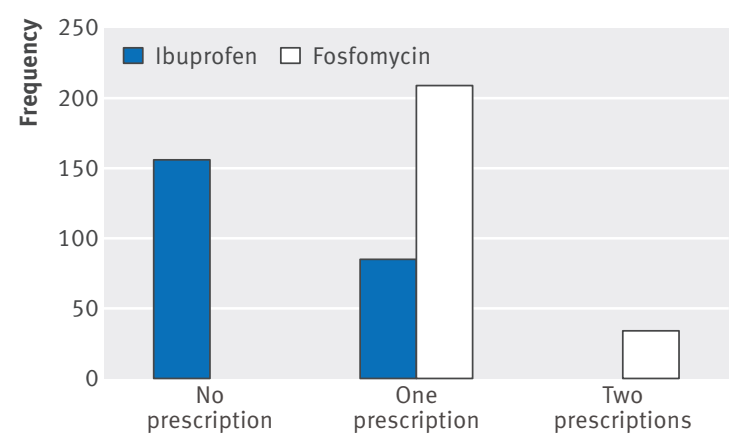

Fig 2 | Total number of antibiotic prescriptions by randomisation group on days $0-28$ (range $0-2$, intention to treat population)

urinary tract infection and 13 for other reasons). This corresponds to an incidence rate reduction of $66.5 \%$ (95\% confidence interval $58.8 \%$ to $74.4 \%$; $\mathrm{P}<0.001$ ), showing a substantial reduction of antibiotics use. Figure 2 shows the distribution of the individual number of courses of antibiotics by randomisation group. Although significantly more women received prescriptions of antibiotics in the follow-up period (21.2\%), the total number receiving antibiotics was lower in the ibuprofen group by $64.7 \%(\mathrm{P}<0.001$, table 2$)$.
As shown in figure 3, the symptom burden sum score decreased in both groups, from six points on day 0 down to less than one point on day seven, on average. Figure 4 shows the corresponding distribution of the symptom burden area under the curve by randomisation group. Table 2 shows the unadjusted mean symptom burden. With an area under the curve ratio of $140 \%$ $\left(\right.$ area $_{\text {ibu }} /$ area $_{\text {fos }}=140.5 \%$, 95\% confidence interval 125.4\% to $157.3 \%$ ) calculated by the adjusted analysis of log disease burden, the non-inferiority margin of $125 \%$ was significantly exceeded. We can therefore reject the hypothesis of non-inferiority. The reported effects were essentially the same when we restricted analyses to the per protocol population.

\section{Secondary outcomes}

All secondary outcomes related to symptoms favoured the fosfomycin group. On days 0-4, the total symptom burden (area under the curve) in the ibuprofen group was significantly higher than in the fosfomycin group. The same results were shown when we considered symptoms individually: for each symptom (dysuria, frequency/ urgency, abdominal pain) a higher symptom burden on days 0-7 was seen in the ibuprofen group (table 2).

With a mean duration of 5.6 days, symptoms lasted about a day longer in the ibuprofen group. On day

Table 2 | Summary of primary and key secondary outcomes in women with uncomplicated urinary tract infection randomised to ibuprofen or fosfomycin. Figures are numbers (percentage) of women unless stated otherwise

\begin{tabular}{|c|c|c|c|c|}
\hline & Ibuprofen $(n=241)$ & Fosfomycin $(n=243)$ & $\%$ mean difference $(95 \% \mathrm{Cl})$ & $P$ value \\
\hline \multicolumn{5}{|l|}{ Primary endpoints } \\
\hline \multicolumn{5}{|l|}{ Women who received antibiotics: } \\
\hline Total & $85(35)$ & $243(100)$ & $-64.7(-70.7$ to -58.7$)$ & $<0.001$ \\
\hline By randomisation & $0(0)$ & $243(100)$ & -100 & - \\
\hline During follow-up (all)* & $85(35)$ & $34(14)$ & $21.2(13.8$ to 28.7$)$ & $<0.001$ \\
\hline During follow-up (for UTI) & $75(31)$ & $30(12)$ & 18.8 (11.6 to 25.9) & $<0.001$ \\
\hline Mean (SD) symptom burden day 0-7† & $17.3(11.0)$ & $12.1(8.2)$ & $5.3(3.5$ to 7.0$)$ & $<0.001$ \\
\hline \multicolumn{5}{|l|}{ Secondary endpoints } \\
\hline \multicolumn{5}{|l|}{ Adverse events in patients: } \\
\hline Patients reporting serious adverse events $\neq$ & $4(2)$ & $0(0)$ & $1.7(0.0$ to 3.3$)$ & 0.06 \\
\hline Serious adverse events probably drug related & $1(0.4)$ & $0(0)$ & $0.4(-0.4$ to 1.2$)$ & 0.32 \\
\hline Patients reporting adverse events $\ddagger$ & $42(17)$ & $57(24)$ & $-6.0(-13.2$ to 1.1$)$ & 0.12 \\
\hline \multicolumn{5}{|l|}{ Relapses/complications: } \\
\hline All recurrent UTI until day 28 & $27(11)$ & $34(14)$ & $-2.8(-8.7$ to 3.1$)$ & 0.41 \\
\hline Early relapse of symptoms (up to day 14$) \S$ & $13(5)$ & $7(3)$ & $2.5(-1.0$ to 6.1$)$ & 0.18 \\
\hline Recurrence of UTI (day 15-28)§ & $14(6)$ & $27(11)$ & $-5.3(-10.2$ to -0.4$)$ & 0.049 \\
\hline Pyelonephritis§ & $5(2)$ & $1(0.4)$ & $1.7(-0.3$ to 3.6$)$ & 0.12 \\
\hline Febrile UTI/ (day 0-7)§ & $3(1)$ & 0 & $1.2(-0.2$ to 2.6$)$ & 0.12 \\
\hline Worsening symptoms (day 0-7)§ & $8(3)$ & $5(2)$ & $1.3(-1.6$ to 4.1$)$ & 0.42 \\
\hline Patients without symptoms day $4 \S$ & $91 / 234(39)$ & $129 / 229(56)$ & $-17.4(-26.4$ to -8.5$)$ & $<0.001$ \\
\hline Patients without symptoms day $7 \mathbf{9}$ & $163 / 232(70)$ & $186 / 227(82)$ & $-11.7(-19.4$ to -4.0$)$ & 0.004 \\
\hline Mean (SD) symptom duration after randomisation (days) & $5.6(2.2)$ & $4.6(2.2)$ & $0.98(0.59$ to 1.38$)$ & $<0.001$ \\
\hline Mean (SD) symptom burden day $0-4 \dagger$ & $13.1(7.1)$ & $10.1(5.9)$ & $3.0(1.9$ to 4.2$)$ & $<0.001$ \\
\hline Mean (SD) symptom burden with regard to dysuria day 0-7 & $6.8(4.6)$ & $4.5(3.6)$ & $2.3(1.5$ to 3.0$)$ & $<0.001$ \\
\hline Mean (SD) symptom burden with regard to frequency/urgency day 0-7 & $6.5(4.1)$ & $4.6(3.4)$ & $1.8(1.1$ to 2.5$)$ & $<0.001$ \\
\hline Mean (SD) symptom burden with regard to low abdominal pain day 0-7 & $4.1(4.3)$ & $2.9(3.1)$ & $1.2(0.5$ to 1.8$)$ & 0.001 \\
\hline Mean (SD) activity impairment assessment day 0-7 & $30.3(24.5)$ & $19.5(16.7)$ & 10.8 (7.1 to 14.6$)$ & $<0.001$ \\
\hline
\end{tabular}

$\mathrm{SD}=$ standard deviation; UTI=urinary tract infection.

*Including antibiotic prescriptions for other reasons-for instance, acute bronchitis, otitis.

tDefined as area under curve (AUC) of daily symptom sum scores day 0-7.

$¥$ As rated by patients.

$\S$ As rated by general practitioners.

ISymptom free is defined as symptom sum score $=0$. 


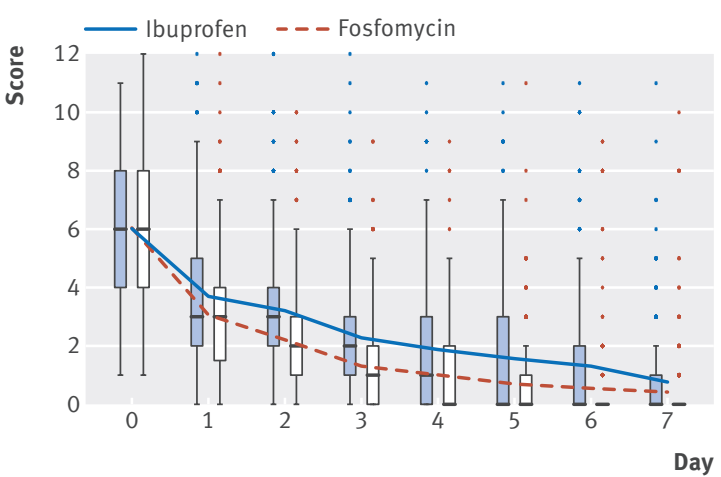

Fig 3 | Symptom sum score for dysuria, frequency/urgency, and low abdominal pain on days $0-7$ by randomisation group (range $0-12$, intention to treat population)

four, $129(56 \%)$ women in the fosfomycin group versus $91(39 \%)$ in the ibuprofen group were symptom free $(\mathrm{P}<0.001$, table 3$)$. Up to day seven these rates increased to $82 \%$ and $70 \%$, respectively $(\mathrm{P}<0.001$, table 3).

Women in the ibuprofen group showed slightly higher scores in impairment of activity-that is, more of their work or regular activities had been impaired by the urinary tract infection (table 4, fig 5). On day seven, $2 \%$ of the women overall still felt impaired most or all the time.

\section{Subgroup analysis}

In women with a positive result on urine culture, an average of 0.49 antibiotic treatment courses per
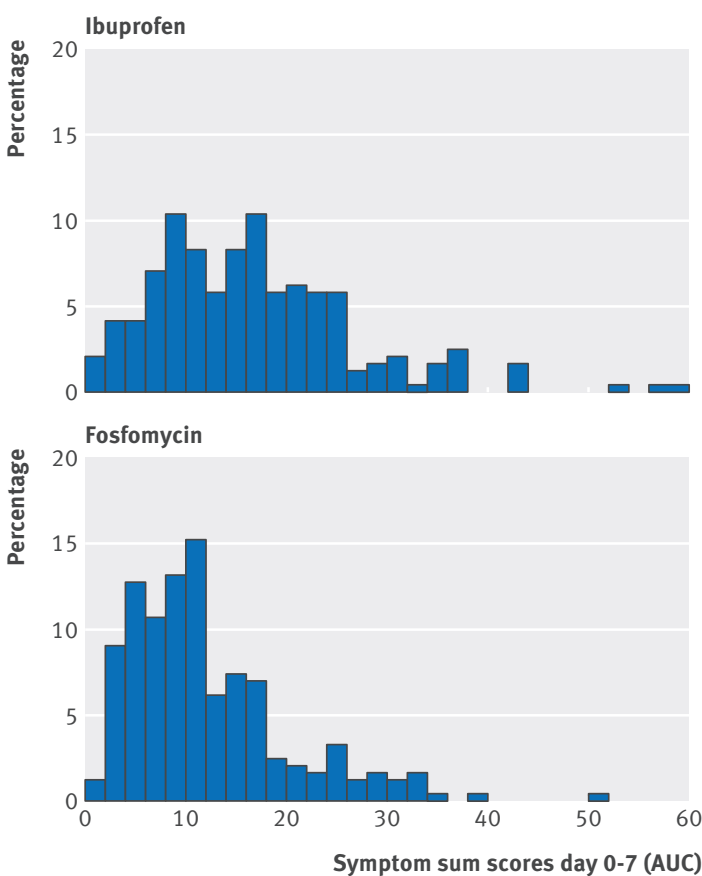

Fig 4 | Distribution of area under curve (AUC) of symptom sum scores for dysuria, frequency/urgency, and low abdominal pain on days $0-7$ by random group (intention to treat population) patient was prescribed in the ibuprofen group versus 1.18 per patient in the fosfomycin group, corresponding to a reduction by $58.5 \%$ (95\% confidence interval $49.8 \%$ to $67.0 \%, \mathrm{P}<0.001)$. In women with a negative result 0.10 antibiotic treatment courses per patient were prescribed in the ibuprofen group versus 1.11 in the fosfomycin group-that is, a reduction by $90.7 \%$ (74.3\% to $99.9 \%, \mathrm{P}<0.001)$. Symptom burden in patients with negative urine culture results did not differ significantly, whereas in patients with positive results, the ibuprofen group again showed a higher burden of symptoms (figs 6 and 7 ). In contrast with the ibuprofen group there was no difference in the symptom burden between those with positive and those with negative results on urine culture in the fosfomycin group (fig 8 ). Table 5 shows differences between women with positive and negative results on urine culture at baseline.

\section{Safety}

The number of complications differed between groups: there were five cases of pyelonephritis in the ibuprofen group and one in the fosfomycin group $(\mathrm{P}=0.12)$. Women who developed pyelonephritis had a higher initial symptom score than average (7.5 versus 6 ). Five women with pyelonephritis consulted their general practitioners with symptoms of upper urinary tract infection (fever, loin tenderness) in the first seven days; one at day 16. Figure 9 shows the courses of symptoms in women with pyelonephritis. All women were treated as outpatients and recovered fully.

The rate of recurrent urinary tract infection was comparable in both groups. There were, however, significantly more recurrences after day 14 in the fosfomycin group ( $11 \%$ versus $6 \%$ in the ibuprofen group, $\mathrm{P}=0.049$, table 2). Of these, 5/14 (36\%) women in the ibuprofen group had a prior history of recurrent urinary tract infections compared with 16/27 (60\%) in the fosfomycin group. In contrast, more women in the ibuprofen group had early relapses of symptoms (up to day 14), but the difference was not significant. (5\% versus $3 \%$; $\mathrm{P}=0.18$, table 2).

There were no significant differences between groups in adverse events not related to urinary tract infection. In the fosfomycin group 57/243 women reported 64 adverse events, whereas in the ibuprofen group 42/241 reported 51 adverse events. Gastrointestinal symptoms (nausea, diarrhoea) were reported more often in the fosfomycin group (15 versus 6). Rashes or vaginal thrush were rare (table 6).

Four patients in the ibuprofen group reported serious adverse events that required hospital admission. One (gastrointestinal haemorrhage) was likely to be drug related; the other three were considered to be unrelated to the drugs (table 7). The woman with gastrointestinal haemorrhage turned out to have an undetected stomach ulcer and alcohol induced hepatitis. Because of a misunderstanding and curiosity, and without having symptoms, one woman unblinded herself using the pharmacy emergency number. 
Table 3 | Patients without symptoms* on day 1-7, intention to treat population of women with uncomplicated urinary tract infection randomised to ibuprofen or fosfomycin. Figures are numbers (percentage) of women unless stated otherwise

\begin{tabular}{lllll}
$\begin{array}{l}\text { Day without } \\
\text { symptoms }\end{array}$ & $\begin{array}{l}\text { Ibuprofen } \\
(\mathbf{n}=\mathbf{2 4 1})\end{array}$ & $\begin{array}{l}\text { Fosfomycin } \\
\mathbf{( n = 2 4 3 )}\end{array}$ & $\begin{array}{l}\text { \% mean difference } \\
\mathbf{9 5 \%} \mathrm{Cl})\end{array}$ & P value \\
\hline 1 & $12 / 240(5)$ & $21 / 231(9)$ & $-4.1(-8.7$ to 0.5$)$ & 0.10 \\
\hline 2 & $30 / 237(13)$ & $45 / 230(20)$ & $-6.9(-13.6$ to 0.3$)$ & 0.045 \\
\hline 3 & $57 / 237(24)$ & $101 / 230(44)$ & $-19.9(-28.3$ to -11.5$)$ & $<0.001$ \\
\hline 4 & $91 / 234(39)$ & $129 / 229(56)$ & $-17.4(-26.4$ to -8.5$)$ & $<0.001$ \\
\hline 5 & $118 / 234(50)$ & $152 / 229(66)$ & $-15.9(-24.8$ to 7.1$)$ & 0.001 \\
\hline 6 & $133 / 233(57)$ & $178 / 227(78)$ & $-21.3(-29.6$ to -13.0$)$ & $<0.001$ \\
\hline 7 & $163 / 232(70)$ & $186 / 227(82)$ & $-11.7(-19.4$ to -4.0$)$ & 0.004 \\
\hline
\end{tabular}

*Defined as symptom sum score $=0$.

Table $4 \mid$ Assessment of impairment of activity on day $0-7$, intention to treat population of women with uncomplicated urinary tract infection randomised to ibuprofen or fosfomycin. Figures are mean (SD) scores

\begin{tabular}{lllll}
$\begin{array}{l}\text { AIA sum } \\
\text { score day* }\end{array}$ & Ibuprofen $(n=241)$ & Fosfomycin $(n=243)$ & $\begin{array}{l}\text { \% mean difference } \\
(\mathbf{9 5 \% ~ C I})\end{array}$ & P value \\
0 & $9.6(5.4)(n=241)$ & $8.9(5.5)(n=238)$ & $0.6(-0.3$ to 1.6$)$ & 0.20 \\
\hline 1 & $7.4(5.7)(n=240)$ & $6.8(5.5)(n=231)$ & $0.6(-0.4$ to 1.6$)$ & 0.24 \\
\hline 2 & $5.6(5.4)(n=237)$ & $3.5(4.4)(n=230)$ & $2.1(1.2$ to 3.0$)$ & $<0.001$ \\
\hline 3 & $3.9(4.8)(n=237)$ & $1.9(3.4)(n=230)$ & $2.0(1.3$ to 2.8$)$ & $<0.001$ \\
\hline 4 & $3.2(4.8)(n=234)$ & $1.1(2.9)(n=229)$ & $2.1(1.4$ to 2.8$)$ & $<0.001$ \\
\hline 5 & $2.6(4.7)(n=234)$ & $0.8(2.4)(n=229)$ & $1.8(1.2$ to 2.5$)$ & $<0.001$ \\
\hline 6 & $2.1(4.4)(n=233)$ & $0.6(2.4)(n=227)$ & $1.5(0.8$ to 2.1$)$ & $<0.001$ \\
\hline 7 & $1.6(3.5)(n=232)$ & $0.6(2.3)(n=227)$ & $1.0(0.5$ to 1.6$)$ & $<0.001$ \\
\hline
\end{tabular}

${ }^{*}$ Activity impairment assessment (AIA) sum scores for five items day 0-7 (range 0-20).

\section{Non-participants}

Non-participants reported higher symptom scores than participants at baseline (8.9 versus 6.0). They had symptoms for longer before visiting the practice, had more recurrent urinary tract infections, and felt more impaired in their daily activities (table 8).

\section{Discussion}

Principal findings

While initial symptomatic treatment with ibuprofen reduced the overall number of antibiotic treatment courses in women with uncomplicated urinary tract infection by $67 \%$, compared with immediate antibiotic treatment with fosfomycin, this strategy resulted in higher burden of symptoms and more cases of pyelonephritis.

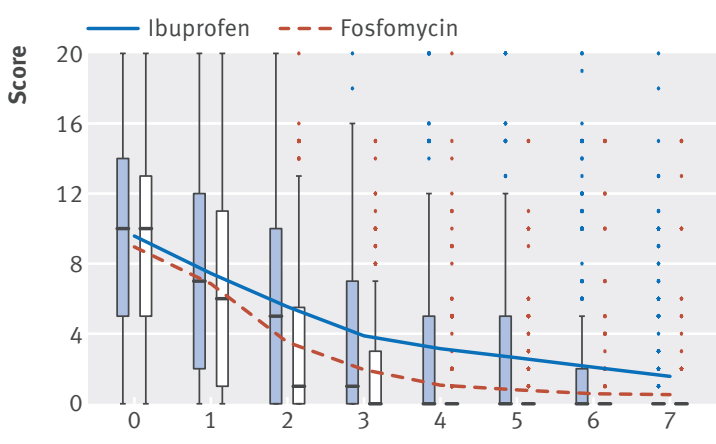

Day

Fig 5 Sum scores for assessment of activity impairment related to urinary tract infection (5 items, range $0-20$, intention to treat population)

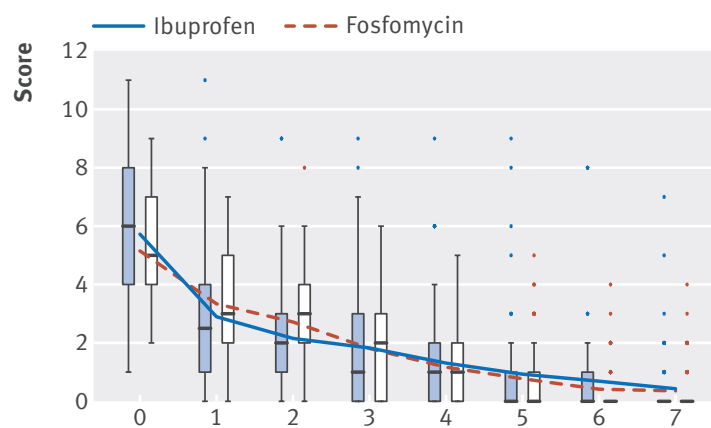

Day

Fig 6 | Symptom sum score for women with negative results on urine culture (subgroup analysis, $n=111$, intention to treat population) on days $0-7$

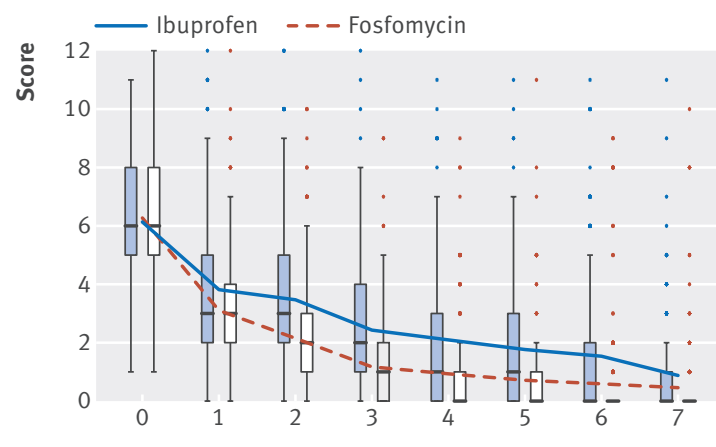

Fig 7 | Symptom sum score for women with positive results on urine culture (subgroup analysis, $n=360$, intention to treat population) on days $0-7$

Two thirds of the women in the ibuprofen group, however, recovered without antibiotic treatment. Within 28 days, $34 \%$ of the ibuprofen group received antibiotic treatment for persistent or worsening symptoms compared with $14 \%$ of the fosfomycin group. These findings are similar to data in the pilot study. ${ }^{10}$ In our trial, lower antibiotic consumption was "bought" at the expense of a higher burden of symptoms until day seven in the ibuprofen group, with an area under the curve ratio of $140 \%$. Although, to our knowledge, ibuprofen has not previously been compared with placebo

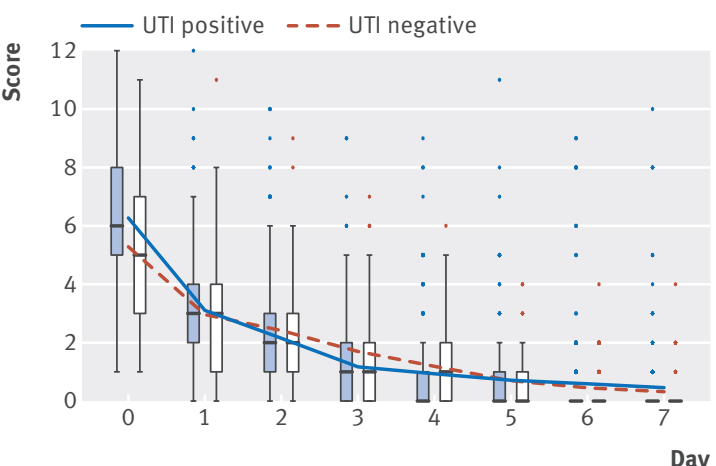

Fig 8 | Symptom sum score for women in fosfomycin group with positive versus negative results on urine culture (subgroup analysis, $\mathrm{n}=\mathbf{2 3 4}$, intention to treat population) on days 0-7 


\begin{tabular}{|c|c|c|c|}
\hline Characteristics & $\begin{array}{l}\text { UTI positive } \\
(\mathrm{n}=360)\end{array}$ & $\begin{array}{l}\text { UTI negative } \\
(\mathrm{n}=111)\end{array}$ & All $(n=471)$ \\
\hline Mean (SD) age (years) & $37.7(14.5)$ & $35.8(14.2)$ & $37.3(14.4)$ \\
\hline Median (IQR) age (years) & $36.0(24.0-49.0)$ & $33.0(23.0-45.0)$ & $35.0(24.0-49.0)$ \\
\hline No (\%) with recurrent UTI & 66/357 (19) & $25 / 110(23)$ & $91 / 467(20)$ \\
\hline \multicolumn{4}{|l|}{ Urinary tract infection symptoms } \\
\hline Mean (SD) symptom duration score & $5.2(2.2)(n=359)$ & $4.7(2.3)(n=111)$ & $5.1(2.2)(n=470)$ \\
\hline Mean (SD) symptom severity score & $6.2(2.3)(n=358)$ & $5.5(2.4)(n=110)$ & $6.0(2.3)(n=468)$ \\
\hline Median (IQR) symptom severity score & $6.0(5.0-8.0)$ & $5.5(4.0-7.0)$ & $6.0(4.0-8.0)$ \\
\hline Mean (SD) dysuria & $2.4(1.0)(n=358)$ & $1.9(1.2)(110)$ & $2.3(1.1)(n=468)$ \\
\hline Median (IQR) dysuria & $3.0(2.0-3.0)$ & $2.0(1.0-3.0)$ & $3.0(2.0-3.0)$ \\
\hline Mean (SD) & $2.4(1.1)(n=358)$ & $2.2(1.0)(n=110)$ & $2.4(1.1)(n=468)$ \\
\hline Median (IQR) frequency/urgency score & $2.0(2.0-3.0)$ & $2.0(1.0-3.0)$ & $2.0(2.0-3.0)$ \\
\hline Mean (SD) lower abdominal pain score & $1.4(1.1)(n=358)$ & $1.3(1.1)(n=110)$ & $1.4(1.1)(n=468)$ \\
\hline Median (IQR) lower abdominal pain score & $1.0(0-2.0)$ & $1.0(0-2.0)$ & $1.0(0-2.0)$ \\
\hline No (\%) with dysuria & $338 / 358(94)$ & $92 / 110(84)$ & 430/468 (92) \\
\hline No (\%) with frequency/urgency & $345 / 358(96)$ & $107 / 110(97)$ & 452/468 (97) \\
\hline No (\%) with low abdominal pain & $251 / 358(70)$ & $80 / 110(73)$ & $331 / 468(71)$ \\
\hline Mean (SD) activity impairment score & $9.4(5.4)(n=358)$ & $8.8(5.7)(n=109)$ & $9.3(5.5)(n=467)$ \\
\hline Median (IQR) activity impairment score & $10.0(5.0-13.0)$ & $9.0(4.0-12.0)$ & $10.0(5.0-13.0)$ \\
\hline \multicolumn{4}{|l|}{ No (\%) with dipstick results: } \\
\hline Leukocytes positive & $316 / 360(88)$ & $82 / 111(74)$ & 398/471 (85) \\
\hline Erythrocytes positive & $292 / 360(81)$ & $73 / 111(66)$ & $365 / 471(78)$ \\
\hline Nitrite positive & $83 / 360(23)$ & $12 / 111(11)$ & $95 / 471(20)$ \\
\hline
\end{tabular}

$\mathrm{SD}=$ standard deviation; $\mathrm{IQR}=$ interquartile range infections. ${ }^{30}$ The pyelonephritis rate in the ibuprofen group in our trial was comparably high (2.1\%). As the study was not powered to detect significant differences for pyelonephritis, it remains unclear whether this is accidental or due to the lack of antibiotic treatment or possibly due to an effect of ibuprofen on the urogenital system. Further research on this particular outcome is needed.

In our trial, ibuprofen was used to ease symptoms of urinary tract infection. An additional antimicrobial effect against $E$ coli has been postulated by Obad and colleagues but could not be confirmed. ${ }^{31}$ Currently the same hypothesis is being investigated by Vik and colleagues. ${ }^{28}$

Recurrent urinary tract infections (day 14-28) were more common in the fosfomycin group. This could be because more women with a history of recurrent urinary tract infection were randomised into the fosfomycin group. Additionally, antimicrobial treatment might result in recurrent urinary tract infections: in a recent trial women with asymptomatic bacteriuria treated with antibiotics had significantly higher rates of subsequent symptomatic urinary tract infection during 12 months' follow-up than untreated women. ${ }^{32}$

For external validity and to comply with routine general practice, we chose a symptomatic approach as recommended in primary care guidelines. ${ }^{7833}$ Therefore, we did not wait for culture results before inclusion and randomisation, and women with negative results on culture remained in the trial. Symptoms and results of urine tests, including the number of negative urine cultures at inclusion, were comparable with other trials in primary care. ${ }^{11163435}$ A subgroup analysis stratified for urine culture results in the ibuprofen group showed significant differences in both co-primary outcomes: women with negative results showed significantly lower symptom burden, and antibiotic use was significantly more reduced in women with positive culture results. Although this was a subgroup analysis, and hence hypothesis generating, these results suggest that urine culture results largely predict the (individual) benefit of antibiotics, though a considerable proportion of those with a positive results did not need antibiotics anyway. Proof of bacterial growth might usefully be included in future antibiotic strategies to curtail unnecessary prescriptions. If sufficiently reliable, point of care tests could reduce the time to targeted treatment compared with conventional culture results, which are available only after several days. A European trial is testing the effectiveness of a point of care test for urinary tract infection with results available within 24 hours in general practices. ${ }^{36}$ Further studies could also provide information on which women with positive test results would require antibiotics and which are likely to recover with symptomatic treatment.

The overall rates of adverse events were comparably low in both groups. The only serious adverse event related to the drug in the ibuprofen group was a gastrointestinal haemorrhage in a woman with alcohol disease, undetected stomach ulcer, and alcohol induced hepatitis. Although rare in otherwise healthy younger women, risks for this serious side effect should be assessed carefully before ibuprofen is prescribed.
Fig 9 | Symptom scores of women with pyelonephritis according to treatment with ibuprofen or fosfomycin on days $0-7$ 


\begin{tabular}{|c|c|c|}
\hline Classification* & Ibuprofen $(n=241)$ & Fosfomycin $(n=243)$ \\
\hline Certain infections and parasitic diseases-such as unspecified viral infection, worms & 3 & 0 \\
\hline Diseases of blood and blood forming organs-such as anaemia & - & 1 \\
\hline Endocrine, nutritional and metabolic diseases_such as goitre & 1 & - \\
\hline Mental and behavioural disorders-such as grief, psychosomatic disorders & 2 & 3 \\
\hline Diseases of the nervous system-such as headache, migraine & 3 & 2 \\
\hline Diseases of the genitourinary system - such as vaginal thrush, local infection & 3 & 1 \\
\hline Diseases of eye and ear-such as unspecific eye lid swelling, otitis, tinnitus & 3 & 5 \\
\hline Diseases of circulatory system-such as hypertonia & - & 1 \\
\hline Diseases of respiratory system-such as upper/lower respiratory infections, asthma & 13 & 12 \\
\hline Diseases of digestive system-such as, nausea, diarrhoea, abdominal pain, vomiting & 6 & 15 \\
\hline Diseases of skin - such as allergy, rash, naevi, eczema, photodermatosis & 3 & 4 \\
\hline Diseases of musculoskeletal system-such as back pain, shoulder syndrome, cervical syndrome & 12 & 14 \\
\hline Injuries and other external causes-such as fall, insect bites & 1 & 4 \\
\hline Symptoms not elsewhere classified-such as abnormal transpiration, non-specific symptoms & 1 & 2 \\
\hline Total No of adverse events & 51 & 64 \\
\hline
\end{tabular}

*Classification corresponds to international statistical classification of diseases and related health problems (ICD).

Table 7 | Details of serious adverse events in women with uncomplicated urinary tract infection randomised to ibuprofen or fosfomycin

\begin{tabular}{lllll} 
Diagnosis & Age (years) & $\begin{array}{l}\text { Beginning (days from } \\
\text { randomisation) }\end{array}$ & $\begin{array}{l}\text { Hospital } \\
\text { admission }\end{array}$ & Trial drug \\
Bleeding gastric ulcer & 58 & 22 & Yes & Ibuprofen \\
\hline Cardiac palpitations & 37 & 1 & Yes & Ibuprofen \\
\hline Missed miscarriage* & 18 & 63 & Yes & Ibuprofen \\
\hline Acute appendicitis & 21 & 18 & Yes & Ibuprofen \\
\hline
\end{tabular}

*One woman assigned to ibuprofen group proved to be pregnant despite negative pregnancy test result at inclusion. Both GP and gynaecologist thought miscarriage was not associated treatment drug.

\section{Strength and limitations}

We were able to enrol more than half of all potentially eligible women, ensuring external validity. While the collection of baseline data from non-participants was a strength of our study, comparison showed that inclusion was biased towards patients with less severe symptoms. This could be caused by self selection, with women with more severe symptoms expressing a preference for antibiotics or general practitioners perceiving sicker patients as unsuitable for the trial. ${ }^{37}$ Results therefore have to be interpreted carefully as they might apply only to patients with mild to moderate symptoms rather than all women with an uncomplicated urinary tract infection.

Another limitation was that both symptom score and the resulting measurement of the area under the curve

\begin{tabular}{|c|c|c|c|}
\hline Characteristics & Participants $(n=484)$ & Non-participants $(n=519)$ & P value \\
\hline \multicolumn{4}{|l|}{ Duration of symptoms (days): } \\
\hline$<1$ & $99 / 480(20)$ & 108/511 (21) & 0.84 \\
\hline $1-2$ & $184 / 480(38)$ & 147/511 (29) & 0.001 \\
\hline$>2-7$ & 148/480 (31) & 192/511 (38) & 0.03 \\
\hline$>7$ & 49/480 (10) & 64/511 (13) & 0.25 \\
\hline $\begin{array}{l}\text { Mean (SD) symptom severity at } \\
\text { baseline }\end{array}$ & $6(2.3)$ & $8.9(2.7)$ & $<0.001$ \\
\hline $\begin{array}{l}\text { Mean (SD) activity impairment } \\
\text { assessment at baseline }\end{array}$ & $9.3(5.5)$ & $14.4(5.5)$ & $<0.001$ \\
\hline Recurrent UTI & 96/479 (20.0) & $127 / 481(26.4)$ & 0.02 \\
\hline
\end{tabular}

$\mathrm{SD}=$ standard deviation; UTI=urinary tract infection. were not validated, and the relevance for affected patients was not formally proved. In our pilot study the primary outcome had been symptom resolution at day 4. This did not adequately feature the severity of symptoms or their duration beyond day 4. We therefore chose the area under the curve of symptom sum score up to day 7 as a pragmatic comprehensive outcome that summarises the aspects of symptom severity and duration relevant to patients, instead of focusing on a single aspect at a prespecified point in time. The validated disease specific activity impairment score showed similar courses.

To further improve outcome measures in future trials, patients' experiences and preferences have to be assessed carefully. Patients should be involved in the development of scores and outcomes. As shown in previous trials, the strategy of delayed prescription could be a welcome and safe alternative for patients who are willing to try treatment other than with antibiotics but want to avoid a reconsultation. This strategy did not reduce antibiotic consumption as effectively as in our trial, but the rate of complications was lower. ${ }^{38}$

The higher rate of pyelonephritis and the fact that more women experienced worsening symptoms and febrile urinary tract infection when treated with ibuprofen needs to be assessed further in controlled trials with larger patient samples. Furthermore, factors associated with successful symptomatic treatment-particularly in patients with positive results on urine culture-should be analysed to develop a decision aid as a tool for physicians and patients to assess whether to treat symptomatically or not.

\section{Conclusions}

We have to reject the hypothesis of non-inferiority of initial symptomatic treatment, and we cannot generally recommend the ibuprofen first approach. This treatment option, however, can be discussed with women with mild to moderate symptoms in a shared decision making approach or within a strategy of delayed prescription. Future research is needed to identify patients for whom 
symptomatic treatment is sufficient as it has the potential to considerably reduce the number of antibiotic prescriptions for women with mild to moderate symptoms of urinary tract infection, in particular those with negative results on culture.

We thank all participating women and all general practice investigators and their teams (Drs Albrecht, Annweiler, Baumgarten, Beverungen, Borchers, Böttcher, Brockstedt, Brucker, Buck, Coutelle, Dickow, Dockhorn, Egidi, Ertel, Falkenstein, Fleige, Gemen, Gerken, Glatzel, Gosewisch, Heiken, Hermann, Hiller, Hilgenberg, Holm, Keske, Kiwit-Putzer, Klinger-Bültemann, Knöpfel, Koch, Köhler, Löber, Lückerath, Meggers, Meier-Ahrens, Menke, Müller, Neidhardt, Preiskorn, Rötterink, Schaper, Schelp, Schmiemann, Schmitz, Seker, Stegemann, Wilde, Zedler, Zimny). We are grateful to our dedicated research nurses H Schneider-Rudt, C Tomala, J Westphal, and K Jürgensen-Muziol. We also thank Ludwig Balzer for support in the statistical analysis and Frank Sullivan for checking of some English language points.

Data Safety and Monitoring Board: Norbert Donner-Banzhoff (Philipps-University Marburg, Department of General Practice, Preventive and Rehabilitation Medicine), Jürgen Brockmöller (University Medical Centre Göttingen, Clinical Pharmacology), Helmut Eiffert (University Medical Centre Göttingen, Institute for Medical Microbiology), Wilhelm Niebling (University Medical Centre Freiburg, Division of General Practice), Andreas Sönnichsen (Institute for General Practice and Family Medicine, Faculty of Health, Witten/ Herdecke), Hans-Joachim Trampisch (Ruhr-University Bochum, Department of Medical Informatics and Epidemiology).

Contributors: IG and JB contributed equally to the manuscript and share first authorship. All authors conceived the concept and designed and supervised to study; acquired, analysed, and interpreted the data; and drafted and revised the manuscript. KW carried out the statistical analysis. EH-P obtained funding. The Institute for Clinical Research, Göttingen (IFS), pharmacy of the Charité (Berlin), medical laboratory AMEDES Holding AG (Göttingen) contributed administrative, material, and technical support. EH-P and IG are guarantors.

Funding: The trial was funded by the German Federal Ministry of Education and Research (BMBF) No 01KG1105. The funder had no role in trial design, data collection, analysis, or reporting.

Competing interests: All authors have completed the uniform disclosure form at www.icmje.org/coi disclosure.pdf and declare: support from the German Federal Ministry of Education and Research for the submitted work; no financial relationships with any organisations that might have an interest in the submitted work in the previous three years; and no other relationships or activities that could appear to have influenced the submitted work. The University of Göttingen was responsible for the initiation, quality control and financial management the clinical study but did not participate in the collection, clinical project management, analysis, and interpretation of data.

Ethical approval: This study was approved by the ethics committee of Hannover Medical School (No 5986M). All participants provided written informed consent.

Transparency: The guarantors affirm that the manuscript is an honest, accurate, and transparent account of the study being reported; that no important aspects of the study have been omitted; and that any discrepancies are disclosed.

Data sharing: Patient level data are available on reasonable request from the corresponding author. Patient consent was not obtained but the presented data are anonymised and risk of identification is low.

This is an Open Access article distributed in accordance with the Creative Commons Attribution Non Commercial (CC BY-NC 3.0) license, which permits others to distribute, remix, adapt, build upon this work non-commercially, and license their derivative works on different terms, provided the original work is properly cited and the use is non-commercial. See: http://creativecommons.org/licenses/ by-nc/3.0/

1 Hooton TM. Clinical practice. Uncomplicated urinary tract infection. N Engl/ Med 2012;366: 1028-37. doi:10.1056 NEIMcp1104429 22417256

2 Ong DS, Kuyvenhoven MM, van Dijk L, Verheij TJ. Antibiotics for respiratory, ear and urinary tract disorders and consistency among GPs. J Antimicrob Chemother 2008;62: 587-92. doi:10.1093/jac/ dkn230 18544602

3 Centers for Disease Control and Prevention. Antibiotic Resistance Threats in the United States, 2013. www.cdc.gov/drugresistance/ threat-report-2013/pdf/ar-threats-2013-508.pdf.
4 World Health Organization. Antimicrobial resistance: Global Report on Surveillance 2014 2014. http://apps.who.int/iris/ bitstream/10665/112642/1/9789241564748 eng.pdf.

5 Foxman B. Urinary tract infection syndromes: occurrence, recurrence, bacteriology, risk factors, and disease burden. Infect Dis Clin North Am 2014;28: 1-13. doi:10.1016/j.idc.2013.09.003 24484571

6 Donnan PT, Wei L, Steinke DT. Presence of bacteriuria caused by trimethoprim resistant bacteria in patients prescribed antibiotics: multilevel model with practice and individual patient data. BMJ 2004:328: 1297 doi:10.1136/bmj.328.7451.129715166067

7 Epidemiology, diagnostics, therapy and management of uncomplicated bacterial community acquired urinary tract infection in adults: German S3-Guideline 2010. http://www.awmf.org/leitlinien/ detail/ll/043-044.html.

8 The Dutch College of General Practitioners. Urineweginfecties M05 2013. https://www.nhg.org/standaarden/samenvatting/ urineweginfecties.

9 Christiaens TCM, De Meyere M, Verschraegen G, Peersman W, Heytens S, De Maeseneer JM. Randomised controlled trial of nitrofurantoin versus placebo in the treatment of uncomplicated urinary tract infection in adult women. BrJ Gen Pract 2002;52: 729-34. 12236276

10 Bleidorn J, Gágyor I, Kochen MM, Wegscheider K, HummersPradier E. Symptomatic treatment (ibuprofen) or antibiotics (ciprofloxacin) for uncomplicated urinary tract infection?--results of a randomized controlled pilot trial. BMC Med 2010;8: 30 doi:10.1186/1741-7015-8-30 20504298

11 Little P, Moore MV, Turner S. Effectiveness of five different approaches in management of urinary tract infection: randomised controlled trial. BMJ 2010;340: c199. doi:10.1136/bmi.c199 20139214

12 Patrick DM, Hutchinson J. Antibiotic use and population ecology: how you can reduce your "resistance footprint". CMA/ 2009;180: 416-21. doi:10.1503/cmaj.08062619221355

13 Butler CC, Dunstan F, Heginbothom M. Containing antibiotic resistance: decreased antibiotic-resistant coliform urinary tract infections with reduction in antibiotic prescribing by general practices. Br J Gen Pract 2007:57: 785-92. 17925135

14 Gyssens IC. Antibiotic policy. Int J Antimicrob Agents 2011;38(Suppl): 11-20. doi:10.1016/j.ijantimicag.2011.09.002 22018989

15 Gottesman BS, Carmeli Y, Shitrit P, Chowers M. Impact of quinolone restriction on resistance patterns of Escherichia coli isolated from urine by culture in a community setting. Clin Infect Dis 2009;49: 86975. doi:10.1086/60553019686074

16 Ferry SA, Holm SE, Stenlund H, Lundholm R, Monsen TJ. The natural course of uncomplicated lower urinary tract infection in women illustrated by a randomized placebo controlled study. Scand J Infect Dis 2004;36: 296-301. doi:10.1080/0036554041001964215198188

17 Knottnerus B], Geerlings SE, Moll van Charante EP, ter Riet G. Women with symptoms of uncomplicated urinary tract infection are often willing to delay antibiotic treatment: a prospective cohort study. BMC Fam Pract 2013:14: 71 doi:10.1186/1471-2296-14-71 23721260

18 Leydon GM, Turner S, Smith H, Little PUTIS team. Women's views about management and cause of urinary tract infection: qualitative interview study. BM/ 2010;340: c279. doi:10.1136/bmj. c279 20139217

19 Willems CS, van den Broek D’Obrenan J, Numans ME, Verheij TJ, van der Velden AW. Cystitis: antibiotic prescribing, consultation, attitudes and opinions. Fam Pract 2014:31:149-55. doi:10.1093/fampra/ cmt077 24317602

20 Gágyor I, Hummers-Pradier E, Kochen MM, Schmiemann G, Wegscheider K, Bleidorn J. Immediate versus conditional treatment of uncomplicated urinary tract infection - a randomized-controlled comparative effectiveness study in general practices. BMC Infect Dis 2012;12: 146. doi:10.1186/1471-2334-12-146 22742538

21 Loudon K, Treweek S, Sullivan F, Donnan P, Thorpe KE, Zwarenstein M. The PRECIS-2 tool: designing trials that are fit for purpose. BMJ 2015;350: h2147. doi:10.1136/bmj.h2147 25956159

22 Mauch H, Podbielski A, Herrmann M, Gatermann SG, Fünfstück R, Handrick W. Harnwegsinfektionen: Qualitätsstandards in de mikrobiologisch-infektiologischen Diagnostik. 2nd ed. Urban \& Fischer Verlag/Elsevier GmbH 2005.

23 Cals IW, Butler CC, Hopstaken RM, Hood K, Dinant GI. Effect of point of care testing for $\mathrm{C}$ reactive protein and training in communication skills on antibiotic use in lower respiratory tract infections: cluster randomised trial. BM/ 2009:338: b1374. doi:10.1136/bmj. b1374 19416992

24 Linde M, Mellberg A, Dahlöf C. The natural course of migraine attacks. A prospective analysis of untreated attacks compared with attacks treated with a triptan. Cephalalgia 2006;26: 712-21. doi:10.1111/j.1468-2982.2006.01097.x 16686911

25 Hanania NA, Boota A, Kerwin E, Tomlinson L, Denis-Mize K. Efficacy and safety of nebulized formoterol as add-on therapy in COPD patients receiving maintenance tiotropium bromide: Results from a 6-week, randomized, placebo-controlled, clinical trial. Drugs 2009;69: 1205-16. doi:10.2165/00003495-2009690900000519537837 
26 Wild DJ, Clayson DJ, Keating K, Gondek K. Validation of a patientadministered questionnaire to measure the activity impairment experienced by women with uncomplicated urinary tract infection: the Activity Impairment Assessment (AIA). Health Qual Life Outcomes 2005;3: 42. doi:10.1186/1477-7525-3-4216022727

27 Julious SA. Sample sizes for clinical trials with normal data. Stat Med 2004;23: 1921-86. doi:10.1002/sim.178315195324

28 Vik I, Bollestad M, Grude N. Ibuprofen versus mecillinam for uncomplicated cystitis--a randomized controlled trial study protocol. BMC Infect Dis 2014:14: 693. doi:10.1186 s12879-014-0693-y 25516016

29 van Pinxteren B, Knottnerus BJ, Geerlings SE. NHG-Standaard Urineweginfecties (derde herziening). Huisarts Wet 2013:56: 270-80.

30 Falagas ME, Kotsantis IK, Vouloumanou EK, Rafailidis PI. Antibiotics versus placebo in the treatment of women with uncomplicated cystitis: a meta-analysis of randomized controlled trials. / Infect 2009;58: 91-102. doi:10.1016/j.jinf.2008.12.009.19195714

31 Obad J, Šušković J, Kos B. Antimicrobial activity of ibuprofen: new perspectives on an "Old" non-antibiotic drug. Eur J Pharm Sci 2015;71: 93-8. doi:10.1016/j.ejps.2015.02.011 25708941

32 Cai T, Mazzoli S, Mondaini N. The role of asymptomatic bacteriuria in young women with recurrent urinary tract infections: to treat or not to treat?Clin Infect Dis 2012;55: 771-7. doi:10.1093/cid/cis534 22677710

33 Scottish Intercollegiate Guidelines Network. Management of suspected bacterial urinary tract infection in adults 2012. www.sign. ac.uk/pdf/sign88.pdf.
34 Giesen LG, Cousins G, Dimitrov BD, van de Laar FA, Fahey T. Predicting acute uncomplicated urinary tract infection in women: a systematic review of the diagnostic accuracy of symptoms and signs. BMC Fam Pract 2010;11: 78. doi:10.1186/1471-2296-11-78 20969801

35 Baerheim A, Digranes A, Hunskaar S. Equal symptomatic outcome after antibacterial treatment of acute lower urinary tract infection and the acute urethral syndrome in adult women. Scand J Prim Health Care 1999;17: 170-3. doi:10.1080/028134399750002593 10555247

36 Bates J, Thomas-Jones E, Pickles T. Point of care testing for urinary tract infection in primary care (POETIC): protocol for a randomised controlled trial of the clinical and cost effectiveness of FLEXICULTTM informed management of uncomplicated UTI in primary care. BMC Fam Pract 2014;15: 187. doi:10.1186/s12875-014-0187-4 25425162

37 Donovan JL, de

Salis I, Toerien M, Paramasivan S, Hamdy FC, Blazeby JM. The intellectual challenges and emotional consequences of equipoise contributed to the fragility of recruitment in six randomized controlled trials. J Clin Epidemiol 2014;67: 912-20. doi:10.1016/j. jclinepi.2014.03.010 24811157

38 Little P, Stuart B, Hobbs FDDESCARTE investigators. Antibiotic prescription strategies for acute sore throat: a prospective observational cohort study. Lancet Infect Dis 2014;14: 213-9. doi:10.1016/S1473-3099(13)70294-9 24440616

(C) BMJ Publishing Group Ltd 2015 